\title{
Long-Term Survival in Young Women: Hazards and Competing Risks after Thyroid Cancer
}

\author{
Antoinette M. Stroup, C. Janna Harrell, and Kimberly A. Herget \\ Utah Cancer Registry, The University of Utah, 650 Komas Drive, Suite 106B, Salt Lake City, UT 84108, USA \\ Correspondence should be addressed to Antoinette M. Stroup, nan.stroup@hsc.utah.edu \\ Received 28 April 2012; Revised 19 July 2012; Accepted 31 July 2012 \\ Academic Editor: Anne Kirchhoff
}

Copyright ( $) 2012$ Antoinette M. Stroup et al. This is an open access article distributed under the Creative Commons Attribution License, which permits unrestricted use, distribution, and reproduction in any medium, provided the original work is properly cited.

\begin{abstract}
Background. Differentiated thyroid cancers (DTCs) are one of the most common and survivable cancers diagnosed in women. We examine factors associated with long-term survival and competing risks of death in women diagnosed with DTC under the age of $40(<40)$ and aged 40 and older $(40+)$. Methods. SEER data was used to identify DTCs diagnosed in women from 1975 to 2009. We examined overall (OS), disease-specific (DSS), other cancer (OCS), and non-cancer-related (NCS) survival using multivariate Cox proportional hazards modeling. Results. Observed survival was $97.2 \%$ for $<40(n=14,540)$ and $82.5 \%$ for $40+(n=20,513)$. Distant stage ( $\mathrm{HR}=1.96,95 \%$ CI 1.23-3.07), non-Hispanic Black (HR = 2.04, 95\% CI 1.45-2.87), being unmarried (HR $=1.26$, $95 \% 1.03-1.54$ ), and subsequent primary cancers ( $\mathrm{HR}=4.63,95 \%$ CI $3.76-5.71$ ) were significant for OS in women $<40$. Age was an effect modifier for all survival outcomes. Racial disparities in NCS were most pronounced for young non-Hispanic black women ( $\mathrm{HR}=3.36,95 \% \mathrm{CI} 2.17-5.22)$. Women in both age groups were more likely to die from other causes. Conclusions. Age at diagnosis remains one of the strongest prognostic factors for thyroid cancer survival. More directed efforts to ensure effective care for comorbid conditions are needed to reduce mortality from other causes.
\end{abstract}

\section{Introduction}

The age-adjusted incidence of female thyroid cancer from 2005 to 2009 was 17.3 per 100,000 , making it the 5th leading cancer diagnosis among women according to the National Cancer Institute's Surveillance, Epidemiology, and End Results (SEER) Program [1]. Recent reports estimate that thyroid cancer incidence has been increasing significantly at an average annual rate of $6.6 \%$ per year since 1998 [2]. From 2010 to 2012, 113,690 new cases of thyroid cancer are expected to be diagnosed among US women alone [35]. Thyroid cancer is not only one of the leading types of cancer among women; it is also one of the most survivable with current 5 -year relative survival rates as high as $98 \%$ [1]. The high incidence of thyroid cancer combined with its high survival makes it one of the most prevalent cancers in the country among women, contributing an estimated $6 \%$ $(n=376,969)$ to the total female cancer prevalence as of January 1,2009 [1].

The gender, age, race/ethnic, and histological patterns among individuals diagnosed with thyroid cancer have been studied extensively [1, 6-15]. The association of geographic location and socioeconomic status (SES) with the incidence of thyroid cancer is not well studied, but evidence suggests that incidence rates vary by SEER registry $[7,12,13]$ and that individuals who live in areas with high SES or with a high proportion of insured individuals are more likely to have a higher incidence of thyroid cancer $[9,16-18]$.

There is also a growing body of literature evaluating the association of gender, age, race/ethnicity, treatment, and geography (vis-à-vis SEER registry) on long-term thyroid cancer survival $[6,7,11,15,19-21]$. Although the literature is consistent with regard to the association of age and race/ethnicity with survival (i.e., younger women and nonHispanic Whites are less likely to die from their disease $[6,7,10,11,15,20-22])$, treatment effects on survival are less consistent. Some studies report significant survival advantages among patients who receive total thyroidectomy [20] while others have found no survival benefits [22, 23] when compared to patients who do not receive cancerdirected therapies such as surgery and radioactive iodine (RAI) treatment. The only study considering geographic 
variability in thyroid cancer survival found differential 5-year relative survival of anaplastic thyroid cancer by SEER registry [15]. However, authors attributed this to regional differences in diagnostic and surveillance practices and did not report systematic differences between registries for differentiated thyroid cancer (DTC), which is the most common type of thyroid cancer among young women.

Another long-term outcome among young women diagnosed with thyroid cancer is the development of subsequent primary cancers $[14,24-31]$. In the only study that evaluated the risks of subsequent primary cancers among women diagnosed under age 40, Ronckers and colleagues [26] reported that patients diagnosed under the age of 40 had a 39\% increased risk for developing a second malignancy, whereas women diagnosed at age 40 or older had only a $6 \%$ increased risk for developing a second malignancy.

To our knowledge, no studies to date have addressed competing risks of death and the association of geographic and socioeconomic factors with long-term survival among women diagnosed with DTC before the age of 40 . This study describes long-term survival and expands previous research by exploring potential geographic and socioeconomic risk factors. In addition, we explore long-term survival outcomes in the context of competing risks.

\section{Materials and Methods}

2.1. Study Sample. We used the National Cancer Institute's SEER Database [32] to obtain demographic, socioeconomic, geographic, tumor, treatment, and survival information necessary for analysis. The initial sample was restricted to a cohort of women diagnosed from 1975 to 2009 with histologically confirmed papillary (PTC) or follicular (FTC) thyroid cancers residing in one of 9 SEER registries, including the states of Connecticut, Hawaii, Iowa, New Mexico, and Utah and the metropolitan areas of San Francisco, Detroit, Seattle, and Atlanta. Pre-1990 census county-level SES measures were not available in the SEER database, and as a result when examining SES effects, we limited our analysis to women diagnosed from 1992 to 2009 . This, however, allowed us to include three additional SEER registries in the SES analysis: rural Georgia and the metropolitan areas of San Jose and Los Angeles (SEER 12).

Following the methods of Kosary and colleagues [7], we used the International Classification of Disease for Oncology, 3rd Edition (ICD-O-3) [33], primary site definition for thyroid cancer (C73.9) with the following PTC and FTC histology classifications: (a) PTC: M-8050, M-8260, M8340, M-8350, M-8450; (b) FTC: M-8290, M-8330-M-8332. Because our study includes cases diagnosed prior to January 1,2001 , when the ICD-O-3 coding schema was adopted by registries, we excluded 1,482 cases with histologies which were not valid prior to ICD-O-3 implementation (i.e., histologies which did not exist prior to 2001 based on ICDO-2). These histologies include M-8335 and M-8341-M8344 .

Incident PTC and FTC were limited to primary cancers which were either the first and only cancer diagnosed or the first of multiple cancers diagnosed for each individual.
Subsequent primary cancers, which refer to new primary cancers that develop in a person with a history of cancer, were limited to cancers occurring after an initial primary diagnosis of thyroid cancer. Women who were diagnosed with thyroid cancer after other primary cancers were also excluded $(n=$ $3,228)$. Other exclusions were women with unknown age $(n=1)$ or unknown county of residence $(n=3)$ at diagnosis, other or unknown race/ethnicity $(n=557)$, and incident cases that were only reported via death certificates or were diagnosed at autopsy $(n=437)$. With the above inclusion and exclusion criteria, a total of 36,295 women were eligible for the study. However, women with missing or unknown surgical $(n=137)$ or radiation $(n=465)$ treatment or with missing or unknown stage $(n=641)$ were also excluded for ease in interpretation of outcomes or because of small numbers.

2.2. Measures. The primary outcomes for overall survival (OS) and disease-specific survival (DSS) were deaths due to any cause and deaths due to thyroid cancer, respectively. Cause of death was obtained using vital status and cause of death information from the SEER database [32]. All thyroid cancer patients were followed from the date of diagnosis (1975-2009) to December 31, 2009. Deaths from thyroid cancer were defined using the SEER cause-specific death classification system, which assumes that the cause-of-death coding systems are inherently prone to error, leading to misclassification and potential underreporting of causes of death due to cancer [34]. The SEER cause-specific death classification system addresses this potential bias by recoding causes of death, accounting for tumor sequence (i.e., only one tumor or the first of subsequent tumors), site of the original cancer diagnosis, and comorbidities (e.g., AIDS and/or site-related diseases) [34]. Competing risk analysis was conducted using the same vital status and cause of death information to classify deaths from other (nonthyroid) cancers (OCS, other cancer survival) and deaths from all noncancer causes (NCS, noncancer survival).

In addition to age at diagnosis and histological subtype, we evaluated survival outcomes by year of diagnosis, race/ethnicity, marital status, stage and tumor size at diagnosis, metropolitan/nonmetropolitan county designations, county-level SES, SEER registry, and surgical and radiation treatment. These variables have been previously linked to cancer incidence and survival [7-9, 12, 13, 16, 35-37]. Race/ethnicity was defined using a combination of SEER race and the Hispanic identification algorithm (NHIA) developed by the North American Association of Central Cancer Registries. SEER race and NHIA codes were used to classify women into four major groups: non-Hispanic White (NH White), non-Hispanic Black (NH Black), Hispanic, and Asian/Pacific Islander (API). Women of Hispanic origin could be of any race. Marital status at the time of diagnosis was defined as married (married including common law), not married (single, divorced, widowed, or never married), and unknown.

Methodologies for classifying tumor stage and size at diagnosis follow those reported by Aschebrook-Kilfoy et al. [12]. SEER Historic Stage A was used to classify thyroid 
cancers by stage at diagnosis including local (limited to thyroid gland), regional (limited to thyroid gland and surrounding tissues), and distant (metastatic or systemic) stages. Tumor size for thyroid cancers was not collected by SEER registries until 1983 and therefore, was only included in the SEER 12 SES analysis for the cohort diagnosed from 1992 to 2009. Tumor size was grouped into five categories: $<1 \mathrm{~cm}, 1.1-2.0 \mathrm{~cm}, 2.1-4.0 \mathrm{~cm}, 4.0+\mathrm{cm}$, and unknown size.

Geographic variables include SEER registry and countylevel metropolitan/nonmetropolitan designations based on 1974, 1983, 1993, and 2003 Rural-Urban Continuum Codes (RUCCs) developed by the US Department of Agriculture [38]. The RUCC classification scheme delineates metropolitan counties based on the size of their metropolitan area population. Nonmetropolitan counties are delineated based on their level of urbanization and adjacency to metropolitan areas. Because RUCC classification schemas changed over time, we assigned each cancer record a RUCC designation based on the year of diagnosis: diagnosis year 1975 was assigned to RUCC 1974, diagnosis years 1976-1985 were assigned to RUCC 1983, diagnosis years 1986-1995 were assigned to RUCC 1993, and diagnosis years 1996-2009 were assigned to RUCC 2003. All RUCC codes were consolidated and grouped into the two major groups: metropolitan (metro) and nonmetropolitan (nonmetro). Women with unknown county were not assigned RUCC designations and were, therefore, excluded from analyses $(n=3)$.

Socioeconomic status (SES) was derived using countylevel data from the 1990 and 2000 decennial censuses and defined as the percent of persons whose income was below the federal poverty level (\%POV). The SES analysis was limited by the availability of census data as \% $\mathrm{POV}$ is only available in SEER* Stat starting with the 1990 census. Percent poverty was evenly distributed in tertile groups, with higher categories representing higher SES (or low-poverty areas).

Radiation treatment was defined using broad categories of yes or no radiation treatment. Radiation treatment may include internal RAI $\left({ }^{131} \mathrm{I}\right)$ ablation therapy or external beam radiation. Women were grouped into two broad categories for surgical treatment with women who received lobectomies, partial/incomplete thyroidectomies, total thyroidectomies, and other nonspecific cancer-directed surgery classified as having surgical treatment and all others as not having surgical treatment. Radiation and surgical treatment variables were based on SEER coding guidelines which define treatment as cancer-directed first-course treatment administered as either part of the patient's treatment plan or administered up to 12 months following diagnosis [39].

2.3. Data Analysis. We first evaluated demographic, geographic, tumor, treatment, and socioeconomic differences by comparing women residing in the SEER 9 registries diagnosed with DTC from 1975 to 2009 who were under the age of $40(<40)$ to women diagnosed with DTC at age 40 or older $(40+)$ using chi-square tests of association for categorical variables and $t$-tests for continuous variables. To adjust for multiple comparisons and because we expected that small differences between groups would be statistically significant due to large sample sizes, we set significance at probability levels of $P<0.0038$ based on a Bonferroni correction with 13 bivariate associations $(0.05 / 13=P<$ 0.0038) [40].

We generated the Kaplan-Meier survival curves to evaluate long-term OS and DSS by age group $(<40$ versus $40+$ ) and histological subtype (PTC versus FTC) and used multivariate Cox proportional hazards (Cox $\mathrm{PH}$ ) regression to estimate the relative risk (hazard ratio, HR) of death from any cause (OS) and from thyroid cancer (DSS). All models included year of diagnosis, histological subtype, stage at diagnosis, race/ethnicity, marital status, metro/nonmetro county designations, radiation and surgical treatment, and subsequent primary tumors. The first model included thyroid cancers diagnosed from 1975 to 2009. The second model was similar to the first but included SEER registry to evaluate registry-specific differences in OS and DSS. The third model was created specifically to evaluate the association of SES on OS and DSS and, therefore, was limited to thyroid cancers diagnosed from 1992 to 2009 in SEER 12. Because tumor size for thyroid cancer was not collected until 1983, tumor size was only included in the third model. All women were followed from the time of diagnosis to the end of the study period (December 31, 2009), until death or loss to follow up. In the OS models, events were considered as deaths from any cause $(n=4,007)$, and women who were lost to follow up or still alive on December 31, 2009, were censored $(n=31,046)$. In the DSS models, events were considered as deaths from thyroid cancer $(n=1,025)$ as defined by SEER cause-of-death recode. Women who died of other causes $(n=2,982)$ were censored as were those who were lost to follow up or still alive on December 31, 2009.

Competing risks for other causes of death were analyzed using 1975-2009 SEER 9 data and Kaplan-Meier survival curves. Similar multivariate Cox PH regression models were generated for OCS and NCS. For the OCS analysis, women who died from thyroid cancer or other noncancer causes, were lost to follow up, or were still alive at the end of the study period were censored. For the NCS analysis, women who died from cancer, were lost to follow up, or were still alive at the end of the study period were censored.

Log-rank tests were conducted to evaluate differences in the Kaplan-Meier survival curves. The proportional hazards assumption was assessed for age group, histology group, race/ethnicity, stage, marital status, metro/non-metro county designations, and surgery and radiation treatment. Statistically significant differences were determined at the $P<0.05$ level. All analyses were performed using SAS statistical software, version 9.2 (released March 2008).

\section{Results}

Characteristics of women diagnosed with PTC and FTC from 1975 to 2009 by age group are presented in Table 1. A total of 35,053 women met all inclusion criteria, of these $41.5 \%$ ( $n=14,540)$ were diagnosed before the age of 40 (median age at diagnoses was 31 years $)$ and $58.5 \%(n=20,513)$ were diagnosed at the age of 40 or older (median age at diagnosis was 52 years). PTCs represented a large majority of cases $(89 \%)$, and younger women were more likely to be diagnosed 
with PTC (90.9\%) than older women (87.2\%). Seventy-five percent of female DTCs occurred among NH Whites for both age groups. Younger women were less likely to be married at the time of diagnosis (58.3\%) as compared to older women $(66.2 \%)$ and were less likely to be diagnosed with subsequent primary cancers $(6.4 \%)$ than older women $(12.4 \%)$.

Stage, tumor size, and treatment differences between women $<40$ and $40+$ were also significant. Younger women were less likely to be diagnosed with localized disease (60.9\%) than older women $(66.7 \%)$ and were only slightly more likely to have surgery and/or radiation as part of their first course treatment $(99.4 \%$ surgery, $45.8 \%$ radiation) than older women ( $98.5 \%$ surgery, $43.9 \%$ radiation). Associations by type of surgery received (lobectomy, thyroidectomy, or nonspecific surgery) were assessed for a subsample of women diagnosed from 1992 to 2009. We found that younger women were more likely to have thyroidectomies (78.9\% versus $74.4 \%)$ whereas older women were more likely to have lobectomies (23.5\% versus 20.2\%) (results not shown).

Geographic distributions varied significantly by age group, with San Francisco-Oakland, Iowa, Atlanta, and Utah registries contributing more younger cases than older cases, while Connecticut and Detroit contributed more older cases than younger cases. Younger women were slightly more likely to reside in metro counties $(89.4 \%)$ when compared to older women $(88.0 \%)$. County-level \%POV was also significantly different across the two age groups. Younger women were less likely to live in high-poverty counties (lowest SES tertile) (14.0\%) as compared to older women $(15.7 \%)$.

As expected, more deaths were observed for older women $(17.6 \%)$ than younger women $(2.8 \%)$. Median follow-up time for women $<40$ was approximately 11 years and median follow-up time for women $40+$ was shorter at just over 7 years. Observed survival was $97.2 \%$ and $82.5 \%$ for younger and older women, respectively. Log-rank tests revealed significant survival differences between age groups for both PTC and FTC $(P<0.001)$ (Figure 1). Younger women consistently showed better OS and DSS than older women.

To test for proportional hazards, an interaction term between time and key variables was included in the OS and DSS models, including age group, histologic group, race, stage, marital status, metro/non-metro, surgery, radiation, and subsequent cancers. The interaction between time and age group $(<40$ and $40+)$ was significant $(P<0.0001)$, and thus we stratified all multivariate Cox $\mathrm{PH}$ regression models by age group. No other interactions were significant, indicating that proportional hazards assumptions were met for all other variables in our analysis.

3.1. Overall Survival. We found that age at diagnosis modified many of the HRs for death in the OS model as significance and magnitude varied by age group (Table 2). Unlike older women, differences in OS between women who were diagnosed at regional stage and women diagnosed at localized stage were not statistically different in the $<40$ age group. The difference between distant stage and localized stage was significant for both age groups, but was
TABle 1: Characteristics of women diagnosed with differentiated thyroid cancer 1975-2009 (SEER 9) by age group.

\begin{tabular}{|c|c|c|}
\hline Characteristic (chi-sq. $P$ value ${ }^{ \pm}$) & $\begin{array}{c}<40 \text { years } \\
N(\%)\end{array}$ & $\begin{array}{c}40+\text { years } \\
N(\%)\end{array}$ \\
\hline Total $(N=35,053)$ & $14,540(41.5)$ & $20,513(58.5)$ \\
\hline \multicolumn{3}{|l|}{ Age at diagnosis ${ }^{\dagger \dagger \dagger}$} \\
\hline Mean (std dev) & $30.01(6.37)$ & $54.82(11.42)$ \\
\hline Median age at diagnosis & 31 & 52 \\
\hline \multicolumn{3}{|l|}{ Histology $* * *$} \\
\hline Papillary & $13,215(90.9)$ & $17,883(87.2)$ \\
\hline Follicular & $1,325(9.1)$ & $2,630(12.8)$ \\
\hline \multicolumn{3}{|l|}{ Race and ethnicity*** } \\
\hline Non-Hispanic White & $10,901(75.0)$ & $15,428(75.2)$ \\
\hline Non-Hispanic Black & $774(5.3)$ & $1,270(6.2)$ \\
\hline Hispanic & $1,437(9.9)$ & $1,490(7.3)$ \\
\hline Asian/Pacific Islander & $1,428(9.8)$ & $2325(11.3)$ \\
\hline \multicolumn{3}{|l|}{ Marital status*** } \\
\hline Married/Common law & $8,471(58.3)$ & $13,575(66.2)$ \\
\hline Not married & $5,618(38.6)$ & $6,138(29.9)$ \\
\hline Unknown & $451(3.1)$ & $800(3.9)$ \\
\hline \multicolumn{3}{|l|}{ Multiple primary cancers $* * *$} \\
\hline One primary only & $13,616(93.7)$ & $17,973(87.6)$ \\
\hline 1 st of $2+$ primaries & $924(6.4)$ & $2,540(12.4)$ \\
\hline \multicolumn{3}{|l|}{ SEER Historic Stage $A^{* * *}$} \\
\hline Localized & $8,857(60.9)$ & $13,691(66.7)$ \\
\hline Regional & $5,288(36.4)$ & $6,112(29.8)$ \\
\hline Distant & $395(2.7)$ & $710(3.5)$ \\
\hline \multicolumn{3}{|l|}{ Surgical treatment ${ }^{* * *}$} \\
\hline Surgery & $14,457(99.4)$ & $20,202(98.5)$ \\
\hline No surgery & $83(0.6)$ & $311(1.5)$ \\
\hline \multicolumn{3}{|l|}{ Radiation treatment ${ }^{* *}$} \\
\hline Radiation & $6,655(45.8)$ & $9,012(43.9)$ \\
\hline No radiation & $7,885(54.2)$ & $11,501(56.1)$ \\
\hline \multicolumn{3}{|l|}{ Tumor size $(1983+)^{* * *}$} \\
\hline $0-1 \mathrm{~cm}$ & $2,814(22.7)$ & $5,760(31.1)$ \\
\hline $1.1-2 \mathrm{~cm}$ & $4,048(32.6)$ & $5,003(27.0)$ \\
\hline $2.1-4 \mathrm{~cm}$ & $3,450(27.8)$ & $4,144(22.4)$ \\
\hline$>4 \mathrm{~cm}$ & $796(6.4)$ & $1,414(7.6)$ \\
\hline Unknown & $1,315(10.6)$ & $2,203(11.9)$ \\
\hline \multicolumn{3}{|l|}{ SEER registry*** } \\
\hline San Francisco-Oakland & $2,222(15.3)$ & $2,776(13.5)$ \\
\hline Connecticut & $1,745(12.0)$ & $3,013(14.7)$ \\
\hline Detroit & $2,055(5.9)$ & $3,254(15.9)$ \\
\hline Hawaii & $828(5.7)$ & $1,315(6.4)$ \\
\hline Iowa & $1,664(11.4)$ & $2,198(10.7)$ \\
\hline New Mexico & $959(6.6)$ & $1,564(7.6)$ \\
\hline
\end{tabular}


TABle 1: Continued.

\begin{tabular}{lcc}
\hline Characteristic (chi-sq. $P$ value $\left.{ }^{ \pm}\right)$ & $<40$ years & $40+$ years \\
& $N(\%)$ & $N(\%)$ \\
\hline Seattle & $2,161(14.9)$ & $2,901(14.1)$ \\
Utah & $1,576(10.8)$ & $1,662(8.1)$ \\
Atlanta & $1,330(9.2)$ & $1,830(8.9)$ \\
Rural-Urban continuum Designation & & \\
(county at diagnosis $\left.{ }^{\dagger}\right)^{* *}$ & & \\
Metropolitan counties & $12,996(89.4)$ & $18,048(88.0)$ \\
Nonmetropolitan counties & $1,544(10.6)$ & $2,465(12.0)$ \\
Socioeconomic status-\% poverty & & \\
(county at diagnosis ${ }^{\dagger}$ ) 1992+ ${ }^{* *}$ & & \\
Tertile 1 (high poverty/low SES) & $1,330(14.0)$ & $2,426(15.7)$ \\
Tertile 2 & $4,280(44.9)$ & $6,670(43.2)$ \\
Tertile 3 (low poverty/high SES) & $3,920(41.1)$ & $6,350(41.1)$ \\
Vital status** & & \\
Alive & $14,134(97.2)$ & $16,912(82.5)$ \\
Dead & $406(2.8)$ & $3,601(17.6)$ \\
Median follow-up time (years) & 11.42 & 7.25 \\
Range & $0.00-34.92$ & $0.00-34.92$ \\
\hline
\end{tabular}

*** Chi-square $P<0.0001$; ** chi-square $P<0.001$.

${ }^{\dagger \dagger \dagger}$ Satterthwaite's $t$-statistic $=259.37, P<0.0001$.

${ }^{\dagger} N=3$ cases missing county at diagnosis.

${ }^{ \pm}$Significance adjusted for Bonferroni’s correction for 13 bivariate comparisons: $P<0.0038$

substantially lower in women $<40(\mathrm{HR}=1.96,95 \%$ CI $1.23-$ $3.07)$ as compared to women $40+(\mathrm{HR}=4.96,95 \%$ CI $4.40-$ 5.59).

$\mathrm{NH}$ Black women had a significantly higher risk of death as compared to $\mathrm{NH}$ White women in both age groups. However, in younger women, the racial disparity was larger as $\mathrm{NH}$ Black women had double the hazard rate of their $\mathrm{NH}$ White counterparts $(\mathrm{HR}=2.04,95 \%$ CI 1.45-2.87; $40+\mathrm{HR}=1.21,95 \%$ CI 1.07-1.37). The risk for unmarried women as compared to married women was also higher in both age groups, although the effect size was smaller for women $<40(\mathrm{HR}=1.26,95 \% 1.03-1.54)$ as compared to women $40+(\mathrm{HR}=2.14,95 \%$ CI 2.00-2.30 $)$. Younger women who did not have surgery had 3.65 times the hazard rate of death (95\% CI 1.14-11.72) as compared to women in the same age group who had surgery, and again, the magnitude of the association for younger women was smaller when compared to older women $(\mathrm{HR}=4.97$, 95\% CI $4.17-$ 5.93).

Women $<40$ who were diagnosed with subsequent primary cancers had 4.63 times the risk of death $(95 \% \mathrm{CI}$ 3.76-5.71) than women with only one primary cancer of the thyroid. This is nearly 2.8 times higher than the HR for women who were diagnosed at age 40 and older $(\mathrm{HR}=1.67$, 95\% CI 1.55-1.80). Interestingly, women who did not have radiation as part of their first-course treatment did not have a higher risk of death, and this was consistent for both age groups.
3.2. Disease-Specific (Thyroid Cancer) Survival. There were substantially fewer deaths from thyroid cancer among younger women $(n=63,0.4 \%)$ than older women $(n=$ 962, 4.7\%) (Table 2). Histological subtype, race/ethnicity, marital status, and subsequent primary cancers were not significantly associated with thyroid cancer deaths in women $<40$. However, similar to older women, the effect of distant stage at diagnosis is much more pronounced ( $\mathrm{HR}=7.8,95 \%$ 3.98-16.07) for DSS than it was for OS. Radiation treatment remains insignificant for women $<40$, but the effect of not having surgery was substantially larger for DSS as younger women who did not have surgery were 15 times more likely to die from thyroid cancer than women who had surgery ( $\mathrm{HR}=15.14,95 \%$ CI 3.30-69.49).

3.3. Geographic Differences. After controlling for a number of potential risk factors, geographic differences in survival were only significant for OS among older women in nonmetro. areas. These women had a $20 \%$ greater risk of death from any cause as compared to women who lived in metro areas $(\mathrm{HR}=1.20,95 \%$ CI 1.09-1.32) (results not shown). When we evaluated registry-specific differences in OS and DSS between younger and older women, we found strong, significant differences between age groups for all registries as younger women were consistently and significantly at lower risk of death as compared to older women (results not shown). We found little variation in HRs across registries after controlling for other risk factors (results not shown).

3.4. Socioeconomic Status. High county-level poverty (Tertile 1, low SES) was only a significant risk factor for OS among older women, conferring a $24 \%$ increased risk of death as compared to women living in counties with the lowest poverty levels (Tertile 3, high SES) (HR $=1.24$, 95\% CI 1.111.38) (Table 3 ). Area-based poverty was not a significant risk factor for young women diagnosed with thyroid cancer for both OS and DSS.

3.5. Competing Risks. Log-rank tests for, Kaplan-Meier survival curves for OCS and NCS revealed significant survival differences between age groups $(P<0.001)$ as the $<40$ group experienced significantly better survival outcomes for both OCS and NCS (results not shown). Competing risk analysis revealed significantly greater race/ethnic disparities in the NCS model as compared to what we found in the OS model (Figure 2). Disparities were more pronounced for NCS among young NH Black women in both age groups $(<40 \mathrm{HR}=3.36,95 \% \mathrm{CI} 2.17-5.22 ; 40+\mathrm{HR}=1.44,95 \%$ CI 1.24-1.69). We also found elevated risks for NCS among young APIs ( $\mathrm{HR}=1.82,95 \% \mathrm{CI} 1.14-2.88)$ as compared to $\mathrm{NH}$ Whites, which did not exist in OS and DSS models.

The distribution of causes of death by age group is described in Table 4. The largest percentage of deaths were due to non-cancer-related causes, namely, heart disease $(<40$ $21.6 \% ; 40+37.1 \%)$. Although younger women in our study were less likely to be diagnosed with subsequent cancers (Table 2), they had a larger percentage of deaths due to other types of cancers $(n=113,27.8 \%)$ as compared to 
TABLE 2: Multivariate Cox PH regression analyses 1975-2009 (SEER 9*): overall and disease-specific survival by age group.

\begin{tabular}{|c|c|c|c|c|}
\hline \multirow{3}{*}{ Variable } & \multicolumn{2}{|c|}{ Overall survival } & \multicolumn{2}{|c|}{ Disease-specific (thyroid cancer) survival } \\
\hline & $<40$ years & $40+$ years & $<40$ years & $40+$ years \\
\hline & $\operatorname{HR}(95 \% \mathrm{CI})^{\dagger}$ & $\mathrm{HR}(95 \% \mathrm{CI})^{\dagger}$ & $\operatorname{HR}(95 \% \mathrm{CI})^{\dagger}$ & $\mathrm{HR}(95 \% \mathrm{CI})^{\dagger}$ \\
\hline Total & 14,450 & 20,513 & 14,450 & 20,513 \\
\hline Number of deaths (\%) & $406(2.8)$ & $3,601(17.6)$ & $63(0.4)$ & $962(4.7)$ \\
\hline \multicolumn{5}{|l|}{ Histology subtype } \\
\hline PTC & 1.00 (ref.) & 1.00 (ref.) & 1.00 (ref.) & 1.00 (ref.) \\
\hline FTC & $0.93(0.69-1.26)$ & $1.46(1.35-1.57)$ & $1.65(0.83-3.30)$ & $1.68(1.46-1.93)$ \\
\hline \multicolumn{5}{|l|}{ Stage at diagnosis } \\
\hline Localized & 1.00 (ref.) & 1.00 (ref.) & 1.00 (ref.) & 1.00 (ref.) \\
\hline Regional & $1.13(0.91-1.39)$ & $1.56(1.45-1.67)$ & $1.14(0.64-2.04)$ & $3.76(3.21-4.42)$ \\
\hline Distant & $1.96(1.23-3.07)$ & $4.96(4.40-5.59)$ & $7.8(3.98-16.07)$ & $19.80(16.30-24.05)$ \\
\hline \multicolumn{5}{|l|}{ Race/ethnicity } \\
\hline NH white & 1.00 (ref.) & 1.00 (ref.) & 1.00 (ref.) & 1.00 (ref.) \\
\hline NH black & $2.04(1.45-2.87)$ & $1.21(1.07-1.37)$ & $0.59(0.14-2.44)$ & $0.85(0.65-1.12)$ \\
\hline Hispanic & $1.35(0.96-1.90)$ & $0.89(0.77-1.02)$ & $1.10(0.47-2.58)$ & $0.99(0.78-1.24)$ \\
\hline API & $1.35(0.98-1.86)$ & $1.06(0.93-1.15)$ & $0.80(0.32-2.02)$ & $1.00(0.83-1.21)$ \\
\hline \multicolumn{5}{|l|}{ Marital status } \\
\hline Married & 1.00 (ref.) & 1.00 (ref.) & 1.00 (ref.) & 1.00 (ref.) \\
\hline Not married & $1.26(1.03-1.54)$ & $2.14(2.00-2.30)$ & $1.12(0.67-1.86)$ & $2.13(1.86-2.43)$ \\
\hline Unknown & $1.17(0.59-2.30)$ & $1.61(1.31-1.97)$ & $0.67(0.09-5.09)$ & $1.40(0.93-2.10)$ \\
\hline \multicolumn{5}{|l|}{ Rural-Urban Continuum } \\
\hline Metropolitan & 1.00 (ref.) & 1.00 (ref.) & 1.00 (ref.) & 1.00 (ref.) \\
\hline Nonmetropolitan & $0.95(0.69-1.31)$ & $1.2(1.09-1.32)$ & $0.64(0.26-1.61)$ & $1.14(0.95-1.38)$ \\
\hline \multicolumn{5}{|l|}{ Radiation treatment } \\
\hline Yes (radiation received) & 1.00 (ref.) & 1.00 (ref.) & 1.00 (ref.) & 1.00 (ref.) \\
\hline No radiation & $1.06(0.84-1.33)$ & $1.05(0.97-1.13)$ & $0.61(0.35-1.06)$ & $0.66(0.58-0.76)$ \\
\hline \multicolumn{5}{|l|}{ Surgical treatment } \\
\hline Yes (surgery received) & 1.00 (ref.) & 1.00 (ref.) & 1.00 (ref.) & 1.00 (ref.) \\
\hline No surgery & $3.65(1.14-11.72)$ & $4.97(4.17-5.93)$ & $15.14(3.30-69.49)$ & $4.97(3.87-6.39)$ \\
\hline \multicolumn{5}{|l|}{ Subsequent primary cancers } \\
\hline No subsequent primaries & 1.00 (ref.) & 1.00 (ref.) & 1.00 (ref.) & 1.00 (ref.) \\
\hline $1+$ subsequent primaries & $4.63(3.76-5.71)$ & $1.67(1.55-1.80)$ & $1.67(0.86-3.25)$ & $0.56(0.46-0.68)$ \\
\hline Year of diagnosis & $0.99(0.97-1.00)$ & $0.99(0.98-0.99)$ & $0.98(0.94-1.02)$ & $0.97(0.96-0.97)$ \\
\hline
\end{tabular}

TABle 3: Multivariate Cox PH regression analyses 1992-2009 (SEER 12*): overall and disease-specific survival by age group ${ }^{\ddagger}$

\begin{tabular}{|c|c|c|c|c|}
\hline \multirow{3}{*}{ Variable } & \multicolumn{2}{|c|}{ Overall survival } & \multicolumn{2}{|c|}{ Disease-specific (thyroid cancer) survival } \\
\hline & $<40$ years & $40+$ years & $<40$ years & $40+$ years \\
\hline & $\mathrm{HR}(95 \% \mathrm{CI})^{\dagger}$ & $\mathrm{HR}(95 \% \mathrm{CI})^{\dagger}$ & $\operatorname{HR}(95 \% \mathrm{CI})^{\dagger}$ & $\mathrm{HR}(95 \% \mathrm{CI})^{\dagger}$ \\
\hline \multicolumn{5}{|l|}{$\%$ below poverty } \\
\hline Tertile 1 (low SES) & $1.31(0.83-2.08)$ & $1.24(1.11-1.38)$ & $1.21(0.44-3.35)$ & $1.05(0.88-1.26)$ \\
\hline Tertile 2 (med. SES) & $1.37(0.86-2.16)$ & $1.06(0.94-1.18)$ & $0.93(0.31-2.84)$ & $0.96(0.80-1.17)$ \\
\hline Tertile 3 (high SES) & $1.00(\operatorname{Ref})$ & $1.00($ Ref $)$ & $1.00($ Ref $)$ & $1.00(\operatorname{Ref})$ \\
\hline
\end{tabular}

*SEER 12: Connecticut, Hawaii, Iowa, New Mexico, Utah; metropolitan areas of San Francisco, Detroit, Seattle, Atlanta, San Jose, Los Angeles; and rural Georgia.

${ }^{\ddagger}$ All models control for diagnosis year, histological subtype, stage, tumor size, race/ethnicity, marital status, urban/rural continuum, radiation treatment, surgical treatment, and subsequent primary cancers.

${ }^{\dagger} \mathrm{HR}$ : hazard ratio; CI: confidence interval. 


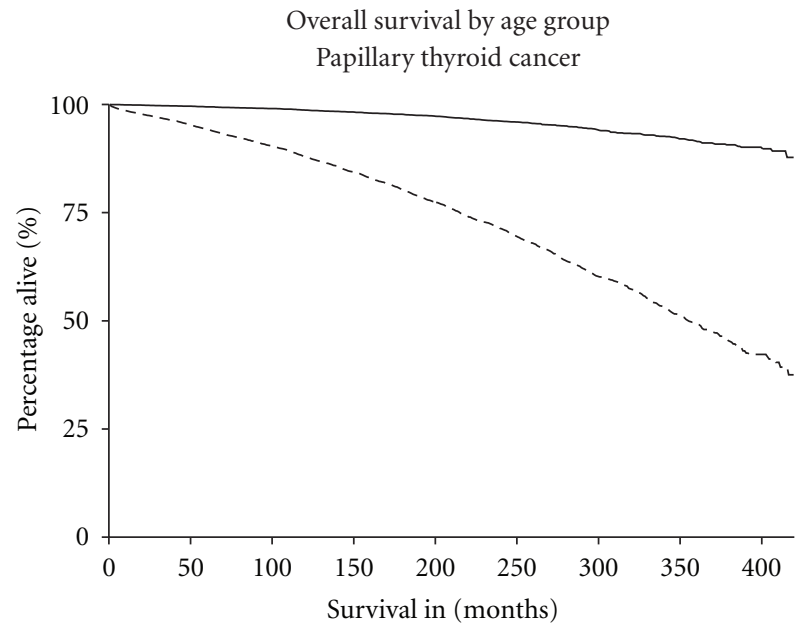

(a)

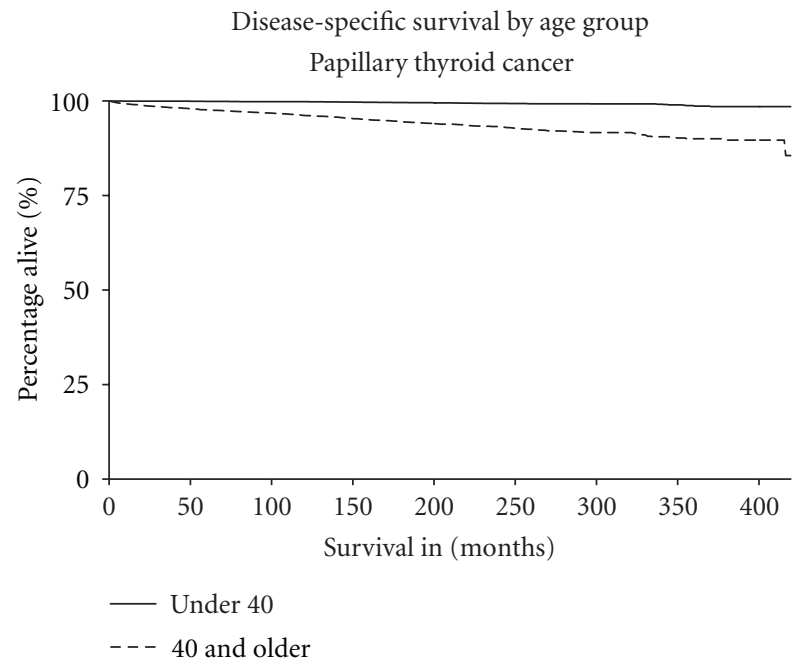

(c)

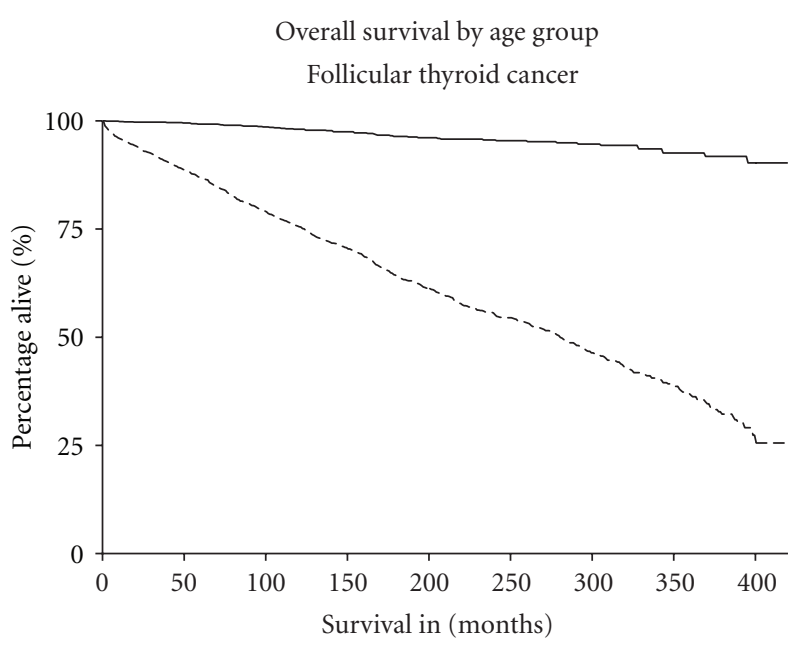

(b)

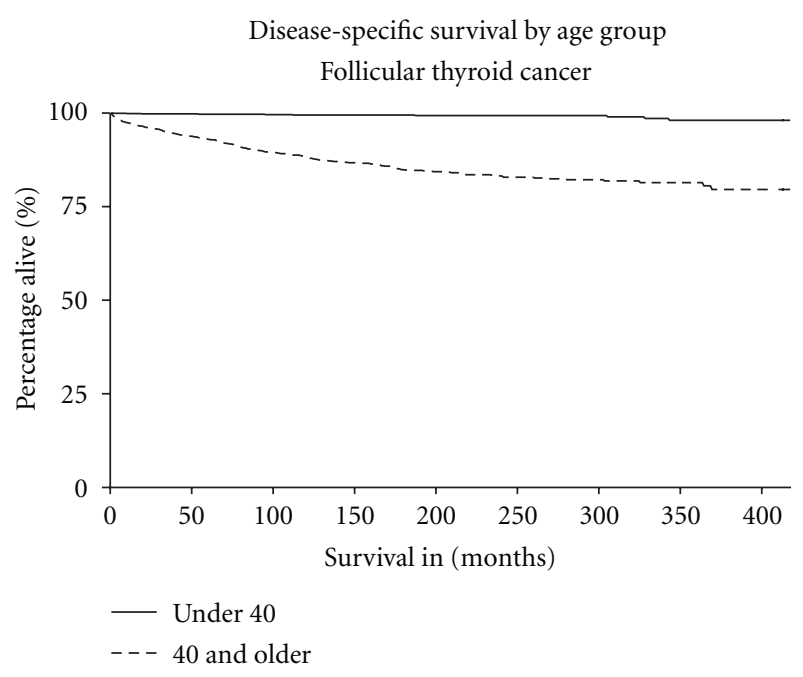

(d)

Figure 1

older women $(n=648,18.0 \%)$. This finding persisted in our multivariate OCS model as being diagnosed with a subsequent primary cancer was the only factor that was significantly associated with deaths from other cancer causes among young women (results not shown).

\section{Discussion}

Rising incidence trends in thyroid cancer and disparities with respect to age and gender have gained considerable attention in recent years [35, 41,42], leading to a growing number of investigations into prognostic clinical and epidemiological determinants of survival from the disease $[6,11,15,20,22]$. This study expands previous research by first describing the long-term survival of women diagnosed with DTC under the age of 40 and exploring potential geographic and socioeconomic risk factors. Then we explore long-term survival outcomes in the context of competing risks. The use of 40 as the age cutoff is a recognized departure from previous thyroid studies $[6,11,14,29,31,43,44]$, save Ronckers et al. [26]; however, there is limited consistency in age grouping across studies. Furthermore, we found compelling statistical evidence vis-à-vis effect modification, which suggests that factors associated with thyroid cancer survival affect women differently when they are diagnosed under the age of 40 as compared to women who are diagnosed at age 40 and older.

Previous studies by Barney et al. [20], Jonklaas et al. [11], and Oyer et al. [6] reported significantly better survival among younger women even after controlling for stage of disease. However, none examined how age at diagnosis might modify stage-specific survival outcomes. In our study, we found that even after controlling for histological subtype and other key demographic and clinical factors, young women with regional stage disease were not significantly different from women with localized disease in terms of thyroid cancer survival. This was not true for women who were diagnosed with regional disease at age 40 and older. 


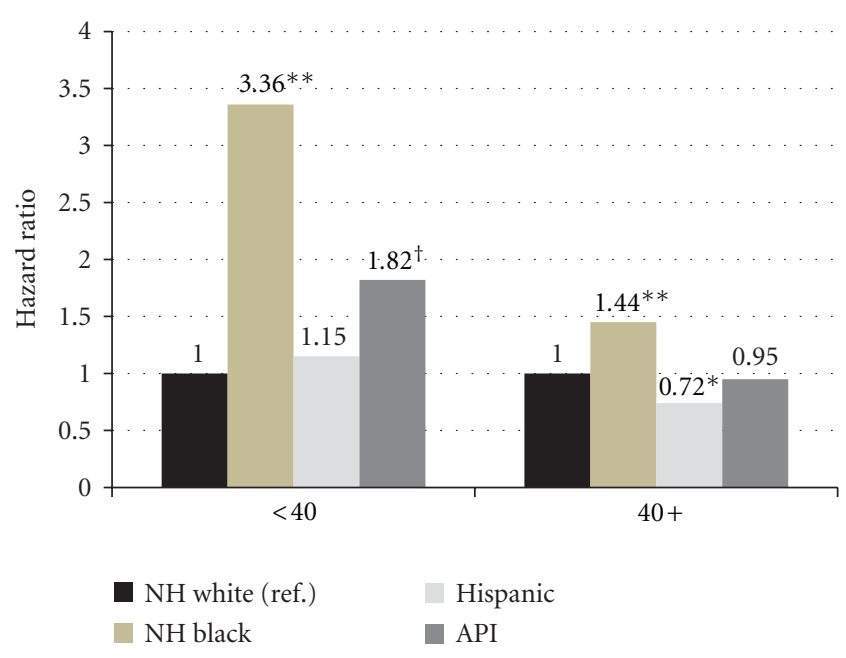

Figure 2: Non-Cancer Related Causes of Death 1975-2009 SEER 9: Race/Ethnicity Hazard Ratios. Model controls for histological subtype, stage, race/ethnicity, marital status, metropolitan/nonmetropolitan county designations, radiation treatment, surgical treatment, and diagnosis year; ${ }^{* *} P<0.0001$; ${ }^{*} P<0.01$; ${ }^{\dagger} P<0.05$.

TABle 4: Distribution of Causes of Death by Age Group: 1975-2009 SEER 9 (Follow-Up through December 31, 2009).

\begin{tabular}{lccc}
\hline Cause of Death & $N$ & \% all cases $\%$ all deaths \\
\hline \multicolumn{4}{c}{$<40$ years $(N=14,450)$} \\
\hline Any Cause & 406 & $2.8 \%$ \\
Thyroid Cancer & 63 & $0.4 \%$ & $15.5 \%$ \\
Other Cancer & 113 & $0.8 \%$ & $27.8 \%$ \\
Other Causes & 176 & $1.2 \%$ & $43.4 \%$ \\
Heart Disease (Rank \#1) & 38 & $21.6 \%^{\dagger}$ & $9.4 \%$ \\
Accidents/Adverse Events (Rank \#2) & 23 & $13.1 \%^{\dagger}$ & $5.7 \%$ \\
Cerebrovascular Disease (Rank \#3) & 17 & $9.7 \%^{\dagger}$ & $4.2 \%$ \\
State DC not available ${ }^{\ddagger}$ & 54 & $0.4 \%$ & $13.3 \%$ \\
\hline \multicolumn{4}{c}{$40+$ years $(N=20,513)$} \\
\hline Any Cause & 3,601 & $17.6 \%$ \\
Thyroid Cancer & 962 & $4.7 \%$ & $26.7 \%$ \\
Other Cancer & 648 & $3.2 \%$ & $18.0 \%$ \\
Other Causes & 1,866 & $9.1 \%$ & $51.8 \%$ \\
Heart Disease (Rank \#1) & 692 & $37.1 \%{ }^{\dagger}$ & $19.2 \%$ \\
Cerebrovascular Disease (Rank \#2) & 221 & $11.8 \%{ }^{\dagger}$ & $6.1 \%$ \\
Chronic Obstructive Pulmonary & 91 & $4.9 \%{ }^{\dagger}$ & $2.5 \%$ \\
Disease (Rank \#3) & 125 & $0.6 \%$ & $3.5 \%$ \\
State DC not available ${ }^{\ddagger}$ & \multicolumn{4}{c}{} \\
\hline
\end{tabular}

${ }^{\dagger} \%$ of other causes of death.

${ }^{\ddagger}$ Censored in all Cox regression models.

We also found that although younger women with distant-stage thyroid cancer were nearly eight times more likely to die from their disease than women of the same age diagnosed with localized disease, the association of late stage diagnosis among older women was much more pronounced, conferring a nearly 20 -fold increase in risk for thyroid cancer death. This is consistent with known age disparities in thyroid cancer survival which led the American Joint Committee on Cancer to incorporate age as a determining factor in the staging of thyroid cancer (i.e., women diagnosed under the age of 45 were assumed to have early stage disease) [45]. This has been criticized for often leading to systematic bias in stage-related outcomes. However, this potential source of bias was not a problem in our study because we used SEER Historic Stage A to define stage at diagnosis, which does not follow AJCC age-specific staging guidelines.

Since staging methodologies are not likely to account for this finding, our results suggest that either younger women may be better equipped to fight their cancer biologically through more robust immune systems or financially through better access to healthcare or that older women have more aggressive tumors which are not adequately captured in the SEER staging or morphology coding systems. Aggressive forms of thyroid cancer are often measured histologically (e.g., anaplastic or medullary thyroid cancers). Our study did not include anaplastic or medullary thyroid cancers which are often more fatal and occur in older women [7]. Aggressive forms of thyroid cancer may also be measured with other tumor features including size [20], extension, nodal involvement, or multifocal attributes. We were limited in our ability to account for other tumor features in our study because of the inconsistency in how these features were reported in SEER from 1975 to 2009 and tumor size was not recorded for thyroid cancer in SEER until 1983 [46]. However, we may have indirectly accounted for tumor size by using the SEER staging system, which includes tumor size in its algorithm. Future research may explore these additional risk factors as well as account for more aggressive forms of papillary thyroid cancers (e.g., diffuse sclerosing and tall cell variants [47]) on the risk of thyroid cancer deaths and use a shorter diagnostic time period when these variables were consistently recorded (e.g., 1988-2009).

Our study also identified additional risk factors which were not examined in previous studies, namely, marital status and the diagnosis of subsequent cancers. It is well established that being married confers a survival benefit for many illnesses, including cancer $[36,37,48]$. We found the same benefit among young women diagnosed with thyroid cancer. The interesting aspect of marital status in our study, however, is that not being married seems to have a larger, negative association among women who were diagnosed after the age of 40. The reason for this finding is not clear but may point to social factors that likely affect older women in different ways (i.e., the absence of social support in combination with competing demands of career and children).

Previous studies have found that younger women have an increased risk of subsequent primary cancers when compared to older women [14, 24-31]. However, we did not see the same pattern in our study. We found that women diagnosed at age 40 or older were more likely to be diagnosed with subsequent cancers than women diagnosed before age 40 in our bivariate analysis. The difference between our study and others is likely due to differences in the 
age cut points. Our multivariate analysis, in fact, showed that the diagnosis of subsequent cancers was significantly associated with poorer overall survival among young women, conferring a nearly 5-fold increase in risk of death from any cause. Our study further showed that the diagnosis of subsequent cancers was the only significant risk factor for other (nonthyroid) cancer deaths, ceteris paribus. Although it is reasonable to hypothesize that women were in fact dying from their subsequent cancer, our study did not explore this relationship. The lack of other significant associations could also be a result of small numbers as only $0.8 \%$ of women who were diagnosed with thyroid cancer before the age of 40 died from another cancer and only $3.2 \%$ of women who were diagnosed with thyroid cancer at age 40 or older died from other cancers. However, this may also suggest that there may be other unmeasured and potentially confounding risk factors that were not accounted for in our study (e.g., comorbidities, family history of cancer, insurance status, healthcare access, etc).

Although we failed to find systematic geographic variations and SES effects on survival in younger women, we know of no other study to date that has evaluated the simultaneous effects of these variables in addition to age and other prognostic factors on thyroid cancer survival. We consider these findings as preliminary because we used SEER registry as a proxy for geography and county-level poverty as a proxy for individual-level SES. Our data are also limited in terms of geographic/demographic scope (i.e., SEER 12) and time (i.e., 1992-2009), which limits the generalizability of our findings. Nevertheless, all registries showed a substantial decrease in risk of death in younger women as compared to older women after controlling for other known risk factors. Therefore, we suggest that despite potential regional/geographic differences in healthcare utilization and access, age remains one of the strongest prognostic factors of thyroid cancer survival. Future research should attempt to describe geographic and SES effects using more precise measures including more granular geographic levels and SES at the individual level.

Previous reports considering racial disparities in thyroid cancer survival are mixed $[10,15]$. Although Hollenbeak et al. [10] reported that African Americans have significantly poorer survival from thyroid cancer as compared to Whites, they attributed the higher risk to higher rates of anaplastic and follicular thyroid cancer types and larger tumors. Yu and colleagues [15] found that for papillary thyroid cancers, Blacks had slightly lower observed 5-year survival (91.6\%) when compared to other race/ethnicity groups which had survival rates between $94 \%$ and $95 \%$. However, Yu et al. [15] did not use multivariate methods to control for potential risk factors. In our study, we found that while substantial racial disparities in long-term overall survival exist between non-Hispanic White and non-Hispanic Black women at any age, the racial disparities were much more pronounced among young women and for other (noncancer) causes of death. The racial disparities for noncancer causes of death were also found among young Asian/Pacific Islander women. These findings provide some evidence to suggest that the racial disparities in survival after thyroid cancer are due to competing risks from comorbid conditions (e.g., heart disease), which tend to be higher in minority populations.

Unfortunately, the low number of deaths from thyroid cancer, particularly for those under the age of 40, leads to poorer precision in the models and wide confidence intervals. Some caution should be used when interpreting the results. Our findings may also have limited generalizability to the larger US population as the SEER 9 and SEER 12 registries represent approximately $10 \%$ and $12 \%[49,50]$ of the US population, respectively. And although SEER registries are geographically diverse, populations within their catchment area may come from more urban areas and have a larger proportion of foreign-born residents [51]. Another limitation of our study was our inability to control for insurance status, which has been associated with cancer incidence $[9,16,17]$, early detection $[52-55]$, and survival outcomes [56-58]. A final caveat to our findings is our inability to control for the entire spectrum of treatment protocols (e.g., chemotherapy, hormone treatment, radiation dosing, etc.) as these data were not available in the SEER database. Future research might include a broader range of treatment modalities and protocols to assess long-term survival outcomes.

\section{Conclusions}

Despite the high overall survival rate for thyroid cancer, there remains significant variability in long-term survival outcomes among young women who are diagnosed with this prevalent disease before the age of 40. This study provides strong evidence that age at diagnosis remains one of the strongest prognostic factors for thyroid cancer survival. Based on our analysis of competing risks, we found that women who were diagnosed with thyroid cancer at any age were more likely to die from other causes, including other cancers, suggesting that young, female thyroid cancer survivors may benefit from more directed efforts to ensure that they receive effective care for comorbid conditions including heart disease and multiple primary cancers to reduce mortality from other causes.

\section{Acknowledgments}

Research was supported by the Utah Cancer Registry, which is funded by Contract no. HHSN261201000026C from the National Cancer Institute's SEER Program with additional support from the Utah State Department of Health and the University of Utah.

\section{References}

[1] N. Howlader, A. M. Noone, M. Krapcho, N. Neyman et al., Eds., "SEER Cancer Statistics Review, 1975-2009 (Vintage 2009 Populations)," http://seer.cancer.gov/csr/1975_2009_ pops09/.

[2] C. Eheman, S. J. Henley, R. Ballard-Barbash et al., "Annual report to the Nation on the status of cancer, 1975-2008, featuring cancers associated with excess weight and lack of 
sufficient physical activity," Cancer, vol. 118, no. 9, pp. 2338 2366, 2012.

[3] American Cancer Society, Cancer Facts \& Figures, 2012, American Cancer Society, Atlanta, Ga, USA, 2012.

[4] American Cancer Society, Cancer Facts \& Figures, 2010, American Cancer Society, Atlanta, Ga, USA, 2010.

[5] American Cancer Society, Cancer Facts \& Figures, 2011, American Cancer Society, Atlanta, Ga, USA, 2011.

[6] S. L. Oyer, V. A. Smith, and E. J. Lentsch, "Reevaluating the prognostic significance of age in differentiated thyroid cancer," Otolaryngology, vol. 147, no. 2, pp. 221-226, 2012.

[7] C. L. Kosary, "Cancer of the thyroid," in SEER Survival Monograph: Cancer Survival Among Adults: U.S. SEER Program, 1988-2001, Patient and Tumor Characteristics, J. L. Young, L. A. G. Ries, G. E. Keel, M. P. Eisner, Y. D. Lin, and M.-J. Horner, Eds., pp. 217-226, National Cancer Institute, SEER Program, Bethesda, Md, USA, 2007, NIH Publication 07-6215.

[8] C. Zhu, T. Zheng, B. A. Kilfoy et al., "A birth cohort analysis of the incidence of papillary thyroid cancer in the united states, 1973-2004," Thyroid, vol. 19, no. 10, pp. 1061-1066, 2009.

[9] L. M. Roche, X. Niu, K. S. Pawlish et al., "Thyroid cancer incidence in New Jersey: time trend, birth cohort and socioeconomic status analysis (1979-2006)," Journal of Environmental and Public Health, vol. 2011, Article ID 850105, 10 pages, 2011.

[10] C. S. Hollenbeak, L. Wang, P. Schneider et al., "Outcomes of thyroid cancer in African Americans," Ethnicity \& Disease, vol. 21, no. 2, pp. 210-215, 2011.

[11] J. Jonklaas, G. Nogueras-Gonzalez, M. Munsell et al., "The impact of age and gender on papillary thyroid cancer survival," The Journal of Clinical Endocrinology \& Metabolism, vol. 97, no. 6, pp. E878-E887, 2012.

[12] B. Aschebrook-Kilfoy, M. H. Ward, M. M. Sabra, and S. S. Devesa, "Thyroid cancer incidence patterns in the United States by histologic type, 1992-2006," Thyroid, vol. 21, no. 2, pp. 125-134, 2011.

[13] B. A. Kilfoy, S. S. Devesa, M. H. Ward et al., "Gender is an age-specific effect modifier for papillary cancers of the thyroid gland," Cancer Epidemiology Biomarkers and Prevention, vol. 18, no. 4, pp. 1092-1100, 2009.

[14] A. P. Brown, J. Chen, Y. J. Hitchcock, A. Szabo, D. C. Shrieve, and J. D. Tward, "The risk of second primary malignancies up to three decades after the treatment of differentiated thyroid cancer," Journal of Clinical Endocrinology and Metabolism, vol. 93, no. 2, pp. 504-515, 2008.

[15] G. P. Yu, J. C. L. Li, D. Branovan, S. McCormick, and S. P. Schantz, "Thyroid cancer incidence and survival in the national cancer institute Surveillance, epidemiology, and end results race/ethnicity groups," Thyroid, vol. 20, no. 5, pp. 465$473,2010$.

[16] B. L. Sprague, S. Warren Andersen, and A. Trentham-Dietz, "Thyroid cancer incidence and socioeconomic indicators of health care access," Cancer Causes and Control, vol. 19, no. 6, pp. 585-593, 2008.

[17] L. G. T. Morris, A. G. Sikora, D. Myssiorek, and M. D. DeLacure, "The basis of racial differences in the incidence of thyroid cancer," Annals of Surgical Oncology, vol. 15, no. 4, pp. 1169-1176, 2008.

[18] T. Haselkorn, L. Bernstein, S. Preston-Martin, W. Cozen, and W. J. Mack, "Descriptive epidemiology of thyroid cancer in Los Angeles County, 1972-1995," Cancer Causes and Control, vol. 11, no. 2, pp. 163-170, 2000.

[19] Y. D. Podnos, D. D. Smith, L. D. Wagman, and J. D. I. Ellenhorn, "Survival in patients with papillary thyroid cancer is not affected by the use of radioactive isotope," Journal of Surgical Oncology, vol. 96, no. 1, pp. 3-7, 2007.

[20] B. M. Barney, Y. J. Hitchcock, P. Sharma, D. C. Shrieve, and J. D. Tward, "Overall and cause-specific survival for patients undergoing lobectomy, near-total, or total thyroidectomy for differentiated thyroid cancer," Head and Neck, vol. 33, no. 5, pp. 645-649, 2011.

[21] M. R. Vriens, W. Moses, J. Weng et al., "Clinical and molecular features of papillary thyroid cancer in adolescents and young adults," Cancer, vol. 117, no. 2, pp. 259-267, 2011.

[22] H. W. Lin and N. Bhattacharyya, "Survival impact of treatment options for papillary microcarcinoma of the thyroid," Laryngoscope, vol. 119, no. 10, pp. 1983-1987, 2009.

[23] L. Davies and G. Welch, "Thyroid cancer survival in the United States: observational data from 1973 to 2005," Archives of Otolaryngology, vol. 136, no. 5, pp. 440-444, 2010.

[24] F. Vaisman, R. Corbo, and M. Vaisman, "Thyroid carcinoma in children and adolescents-systematic review of the literature," Journal of Thyroid Research, vol. 2011, Article ID 845362, 2011.

[25] N. G. Iyer, L. G. T. Morris, R. M. Tuttle, A. R. Shaha, and I. Ganly, "Rising incidence of second cancers in patients with low-risk (T1N0) thyroid cancer who receive radioactive iodine therapy," Cancer, vol. 117, no. 19, pp. 4439-4446, 2011.

[26] C. M. Ronckers, P. McCarron, and E. Ron, "Thyroid cancer and multiple primary tumors in the SEER cancer registries," International Journal of Cancer, vol. 117, no. 2, pp. 281-288, 2005.

[27] A. K. Ying, W. Huh, S. Bottomley et al., "Thyroid cancer in young adults," Seminars in Oncology, vol. 36, no. 3, pp. 258274, 2009.

[28] R. M. Hirosawa, M. Marivo, J. de Moura Leite Luengo et al., "Does radioiodine therapy in patients with differentiated thyroid cancer increase the frequency of another malignant neoplasm?" ISRN Oncology, vol. 2011, Article ID 708343, 2011.

[29] T. C. Sandeep, M. W. Strachan, R. M. Reynolds et al., "Second primary cancers in thyroid cancer patients: a multinational record linkage study," The Journal of Clinical Endocrinology \& Metabolism, vol. 91, no. 5, pp. 1819-1825, 2006.

[30] A. M. Sawka, L. Thabane, L. Parlea et al., "Second primary malignancy risk after radioactive iodine treatment for thyroid cancer: a systematic review and meta-analysis," Thyroid, vol. 19, no. 5, pp. 451-457, 2009.

[31] C. I. Li, M. A. Rossing, L. F. Voigt, and J. R. Daling, "Multiple primary breast and thyroid cancers: role of age at diagnosis and cancer treatments (United States)," Cancer Causes and Control, vol. 11, no. 9, pp. 805-811, 2000.

[32] E. Surveillance and End Results (SEER) Program, "SEER* Stat Database: Incidence-SEER 9 Regs Research Data, Nov 2011 Sub, Vintage 2009 Pops (1973-2009) Katrina/Rita Population Adjustment-Linked To County Attributes-Total U.S., 1969-2010 Counties, National Cancer Institute, DCCPS, Surveillance Research Program, Surveillance Systems Branch," 2012, http://www.seer.cancer.gov/.

[33] A. Fritz, A. Jack, D. M. Parkin et al., International Classification of Diseases for Oncology, World Health Organization, Geneva, Switzerland, 3rd edition, 2000.

[34] N. Howlader, L. A. G. Ries, A. B. Mariotto, M. E. Reichman, J. Ruhl, and K. A. Cronin, "Improved estimates of cancerspecific survival rates from population-based data," Journal of the National Cancer Institute, vol. 102, no. 20, pp. 1584-1598, 2010.

[35] J. D. Cramer, P. Fu, K. C. Harth, S. Margevicius, and S. M. Wilhelm, "Analysis of the rising incidence of thyroid cancer 
using the Surveillance, epidemiology and end results national cancer data registry," Surgery, vol. 148, no. 6, pp. 1147-1153, 2010.

[36] M. S. Rendall, M. M. Weden, M. M. Favreault, and H. Waldron, "The protective effect of marriage for survival: a review and update," Demography, vol. 48, no. 2, pp. 481-506, 2011.

[37] L. Wang, S. E. Wilson, D. B. Stewart, and C. S. Hollenbeak, "Marital status and colon cancer outcomes in US Surveillance, epidemiology and end results registries: does marriage affect cancer survival by gender and stage?" Cancer Epidemiology, vol. 35, no. 5, pp. 417-422, 2011.

[38] USDA Economic Research Service, "Measuring Rurality: Rural-Urban Continuum Codes," 2004, http://www.ers.usda.gov/Briefing/Rurality/RuralUrbCon/

[39] M. B. Adamo, C. H. Johnson, J. L. Ruhl et al., Eds., SEER Program Coding and Staging Manual, vol. 11-5581, National Cancer Institute, Bethesda, Md, USA, 2011.

[40] S. Holm, "A simple sequentially rejective multiple test procedure," Scandinavian Journal of Statistics, vol. 6, pp. 65-70, 1979.

[41] R. Rahbari, L. Zhang, and E. Kebebew, "Thyroid cancer gender disparity," Future Oncology, vol. 6, no. 11, pp. 1771-1779, 2010.

[42] L. Davies, M. Ouellette, M. Hunter, and H. G. Welch, "The increasing incidence of small thyroid cancers: where are the cases coming from?" Laryngoscope, vol. 120, no. 12, pp. 2446 2451, 2010.

[43] P. L. Horn-Ross, A. J. Canchola, H. Ma, P. Reynolds, and L. Bernstein, "Hormonal factors and the risk of papillary thyroid cancer in the California teachers study cohort," Cancer Epidemiology Biomarkers and Prevention, vol. 20, no. 8, pp. 1751-1759, 2011.

[44] B. H.-H. Lang and K. P. Wong, "Risk factors for nonsynchronous second primary malignancy and related death in patients with differentiated thyroid carcinoma," Annals of Surgical Oncology, vol. 18, no. 13, pp. 3559-3565, 2011.

[45] S. H. Tran Cao, L. E. Johnston, D. C. Chang et al., "A critical analysis of the American Joint Committee on Cancer (AJCC) staging system for diffierentiated thyroid carcinoma in young patients on the basis of Surveillance, Epidemiology, and End Results (SEER) registry," Surgery, vol. 152, no. 2, pp. 145-151, 2012.

[46] E. Shambaugh, L. Ries, and J. Young, Extent of Disease New 4-Digit Schemes Codes and Coding Instructions, Demographic Analysis Section Biometry Branch, National Cancer Institute, Bethesda, Md, USA, 1984.

[47] H. S. Kazaure, S. A. Roman, and J. A. Sosa, "Aggressive variants of papillary thyroid cancer: incidence, characteristics and predictors of survival among 43, 738 Patients," Annals of Surgical Oncology, vol. 19, no. 6, pp. 1874-1880, 2012.

[48] J. D. Sammon, M. Morgan, and O. Djahangirian, "Marital status: a gender-independent risk factor for poorer survival after radical cystectomy," British Journal of Urology International. In press.

[49] Surveillance Epidemiology and End Results (SEER) Program, "Number of Persons by Race and Hispanic Ethnicity for SEER Participants (2000 Census Data)," 2012, http://seer.cancer.gov/registries/data.html.

[50] National Cancer Institute and Centers for Disease Control and Prevention, "State Cancer Profiles," 2012, http://statecancerprofiles.cancer.gov/cgi-bin/dictionary.pl\#S .

[51] B. F. Hankey, L. A. Ries, and B. K. Edwards, "The Surveillance, epidemiology, and end results program: a national resource," Cancer Epidemiology Biomarkers and Prevention, vol. 8, no. 12, pp. 1117-1121, 1999.

[52] S. Martin, C. Ulrich, M. Munsell, S. Taylor, G. Lange, and A. Bleyer, "Delays in cancer diagnosis in underinsured young adults and older adolescents," Oncologist, vol. 12, no. 7, pp. 816-824, 2007.

[53] S. A. Denslow, G. Knop, C. Klaus et al., "Burden of invasive cervical cancer in North Carolina," Preventive Medicine, vol. 54, pp. 270-276, 2012.

[54] M. T. Halpern, E. M. Ward, A. L. Pavluck, N. M. Schrag, J. Bian, and A. Y. Chen, "Association of insurance status and ethnicity with cancer stage at diagnosis for 12 cancer sites: a retrospective analysis," The Lancet Oncology, vol. 9, no. 3, pp. 222-231, 2008.

[55] E. M. Ward, S. A. Fedewa, V. Cokkinides, and K. Virgo, "The association of insurance and stage at diagnosis among patients aged 55 to 74 years in the national cancer database," Cancer Journal, vol. 16, no. 6, pp. 614-621, 2010.

[56] S. A. Fedewa, C. Lerro, D. Chase, and E. M. Ward, "Insurance status and racial differences in uterine cancer survival: a study of patients in the National Cancer Database," Gynecologic Oncology, vol. 122, no. 1, pp. 63-68, 2011.

[57] J. Kwok, S. M. Langevin, A. Argiris, J. R. Grandis, W. E. Gooding, and E. Taioli, "The impact of health insurance status on the survival of patients with head and neck cancer," Cancer, vol. 116, no. 2, pp. 476-485, 2010.

[58] A. S. Robbins, A. L. Pavluck, S. A. Fedewa, A. Y. Chen, and E. M. Ward, "Insurance status, comorbidity level, and survival among colorectal cancer patients age 18 to 64 years in the national cancer data base from 2003 to 2005," Journal of Clinical Oncology, vol. 27, no. 22, pp. 3627-3633, 2009. 


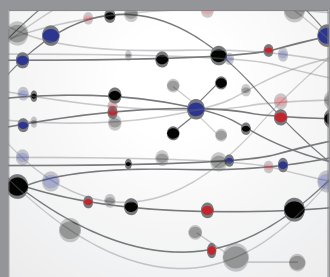

The Scientific World Journal
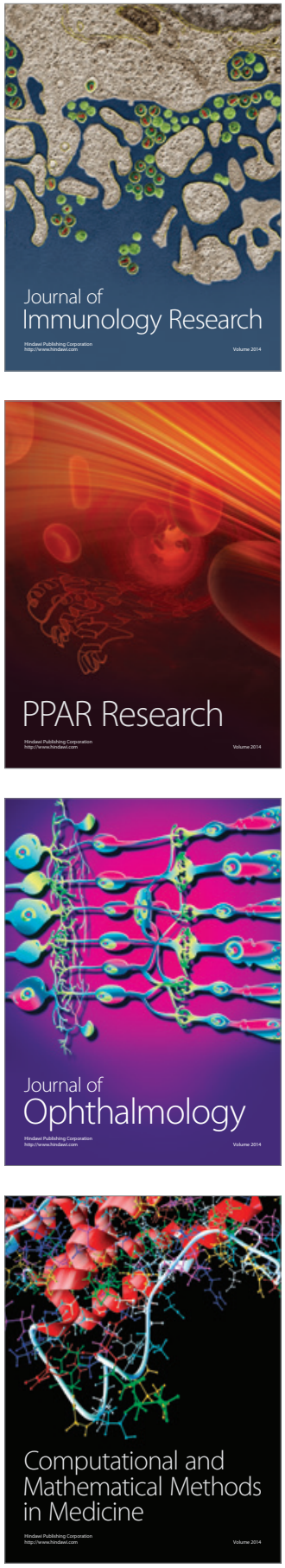

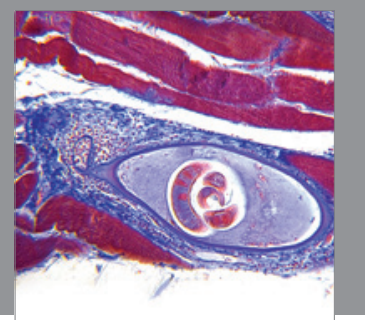

Gastroenterology

Research and Practice
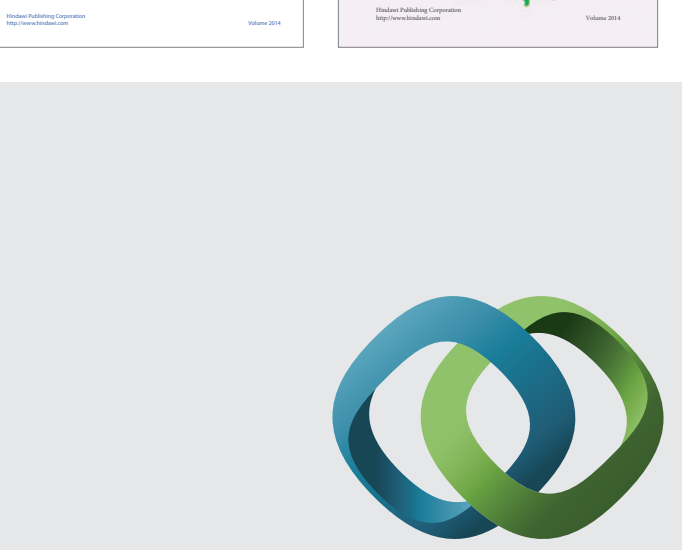

\section{Hindawi}

Submit your manuscripts at

http://www.hindawi.com
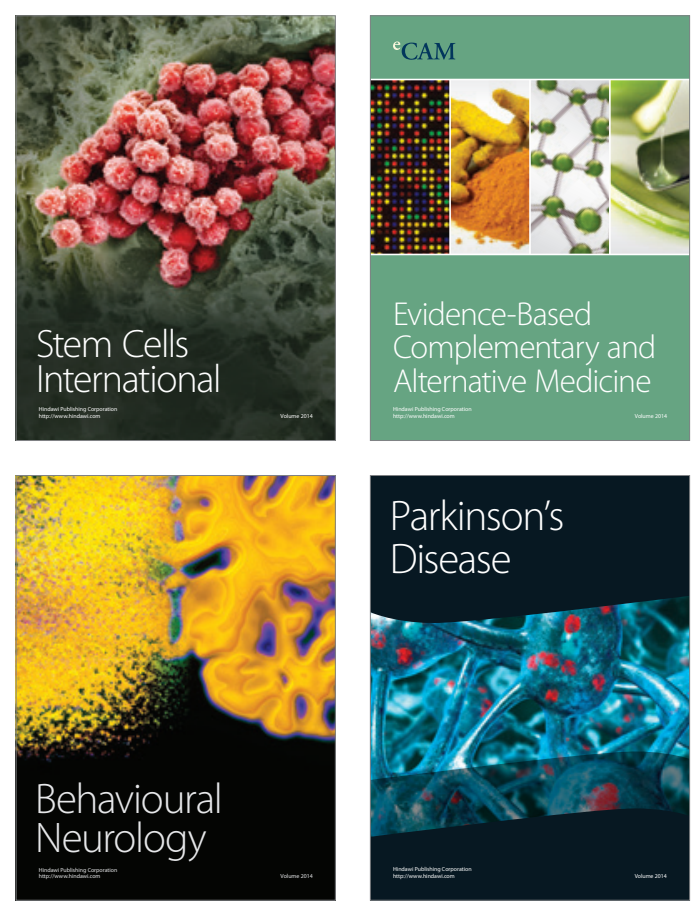

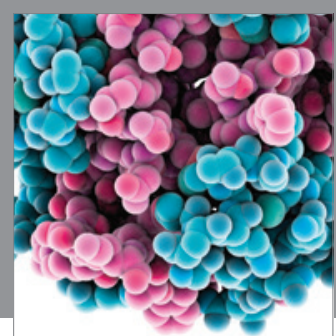

Journal of
Diabetes Research

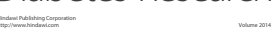

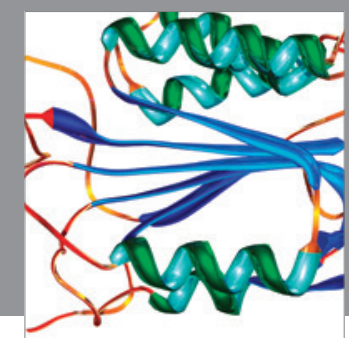

Disease Markers
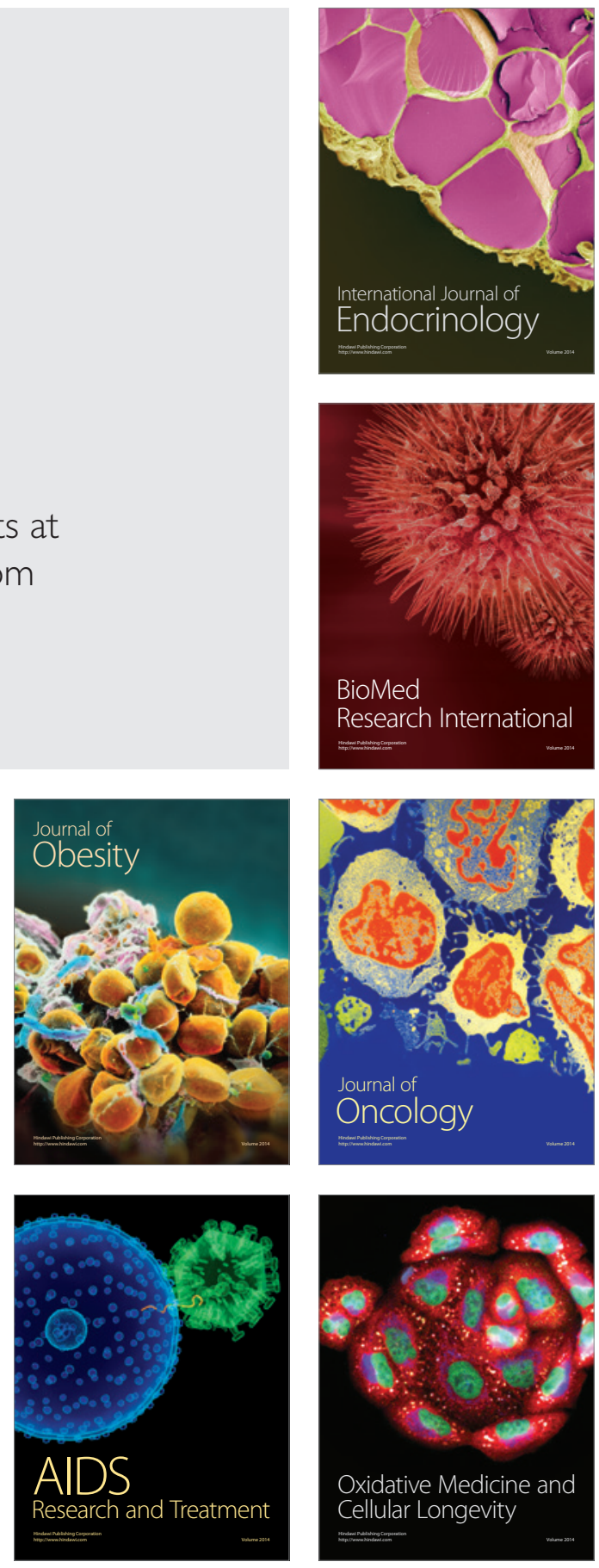